\title{
Algorithms for Computing a Planar Homography from Conics in Correspondence
}

\author{
Juho Kannala, Mikko Salo and Janne Heikkilä \\ Machine Vision Group \\ University of Oulu, Finland \\ \{jkannala, msa, jth@ee.oulu.fi $\}$
}

\begin{abstract}
This paper presents two new algorithms for computing a planar homography from conic correspondences. Firstly, we propose a linear algorithm for computing the homography when there are three or more conic correspondences. In this case, we get an overdetermined set of linear equations and the solution that minimizes the algebraic distance is obtained by the singular value decomposition. Secondly, we propose another algorithm for determining the homography from only two conic correspondences. Unlike the previous algorithms our approach uses only linear algebra and does not require solving high-degree polynomial equations. Hence, the proposed formulation leads to an algorithm that is efficient and easy to implement. In addition, our approach incorporates the computation of the two projective invariants for a pair of conics. These invariants provide a condition for the existence of a homography between the pairs of conics. We evaluate the characteristics and robustness of the proposed algorithms in experiments with synthetic and real data.
\end{abstract}

\section{Introduction}

It is well known that under perspective imaging a plane is mapped to the image by a planar projective transformation. This transformation is called a homography and it is used in many areas of computer vision [3]. Most often the homography between two planes is determined from point correspondences. In this case, at least four correspondences are required and there are several algorithms that have been thoroughly studied [3]. However, in this paper, we study the problem of determining the homography from conic correspondences which is a less explored problem.

In computer vision, conics are widely accepted as one of the most fundamental image features together with points and lines [8]. Like points and lines, conics are invariant under planar projective transformations. This means that a perspective image of a plane conic is still a conic. The motivation for studying the geometry of conics arises from the fact that sometimes point or line correspondences are not available or could be noisy while higher order curves, such as conics, can still be identified robustly. In addition, many common man-made objects contain conics as characteristic features. Though there are fewer articles dealing with conics than those dealing with points and lines, several procedures 
have been proposed for pose estimation, structure recovery and camera calibration that are based on conics, for instance $[2,8,5,9,12]$.

In [2], the projective invariants for pairs of conics were applied in object recognition and an algorithm was developed to determine the relative pose of a scene plane from two conic correspondences. This problem is equivalent to determining the homography between the scene plane and image plane for a calibrated camera but the solution presented in [2] requires quartics that cannot be solved in closed form. Algorithms for projective and metric reconstruction of plane conics from two views were presented in [8] assuming known camera projection matrices. The estimation of epipolar geometry from conic correspondences was discussed in [5] and [6]. In [9], it was shown that, given the epipolar geometry, the homography induced by the plane of a conic can be determined by observing the conic in both views (one correspondence). In camera calibration, the procedure proposed in [12] uses conic correspondences to determine the homography between the calibration plane and its image. However, this approach requires concentric conics.

The closest works to that which we report here are [11] and [7]. In [11] a linear algorithm was proposed for solving the homography from conic correspondences. The algorithm is based on considering conics as points in the projective space $\mathbb{P}^{5}$ and the homography is determined from the corresponding conic-based transformation which is a linear mapping from $\mathbb{P}^{5}$ to $\mathbb{P}^{5}$. This approach requires at least seven correspondences while the linear algorithm presented in this paper requires only three. At the minimum only two conic correspondences are enough for solving the homography as described in [7]. However, the algorithm in [7] has several steps and requires solutions of polynomial equations while we present a direct algorithm using linear algebra.

This paper is organized as follows. In Section 2 we describe the mathematical background. The proposed algorithms, both for the minimal and general case, are described in Section 3. The experiments are reported in Section 4.

\section{Properties of Conics}

A conic is a second-degree curve in the plane. In homogeneous coordinates, a general conic section can be expressed as

$$
\mathbf{x}^{\top} \mathbf{C} \mathbf{x}=0
$$

where $\mathbf{C}$ is a real symmetric $3 \times 3$ matrix containing the conic coefficients [10]. If $\mathbf{C}$ is indefinite and has full rank the conic is an ellipse, hyperbola or parabola. The conic is called degenerate if $\mathbf{C}$ has rank 1 or 2 . The degenerate conics include a single point (rank 2), two lines (rank 2) and a repeated line (rank 1) [3]. Under the point homography $\mathbf{x}^{\prime}=\mathbf{H x}$ a conic $\mathbf{C}$ transforms to $\mathbf{C}^{\prime}=\mathbf{H}^{-\top} \mathbf{C} \mathbf{H}^{-1}$ [3]. All full rank indefinite conics are projectively equivalent to a circle, i.e., every such conic can be transformed to a circle with a homography [3].

Assume that we have a pair of corresponding conics, $\mathbf{C}$ and $\mathbf{C}^{\prime}$, between two planes. The homography $\mathbf{H}$ between the planes is unknown. The conics satisfy

$$
\mathbf{C}^{\prime} \sim \mathbf{H}^{-\top} \mathbf{C H}^{-1}
$$

where $\sim$ denotes equality up to scale. Since there are 8 degrees of freedom in $\mathbf{H}$ (homogeneous $3 \times 3$ matrix) and equation (2) provides only 5 constraints (symmetric matrices 
equal up to scale) it is not possible to solve the homography from one pair of corresponding conics. This is also clear from the above discussion since all indefinite full rank conics are projectively equivalent. However, in general, if we have two conic correspondences, $\mathbf{C}_{1} \leftrightarrow \mathbf{C}_{1}^{\prime}$ and $\mathbf{C}_{2} \leftrightarrow \mathbf{C}_{2}^{\prime}$, we get 10 constraints from which we may solve $\mathbf{H}$.

\section{Algorithms}

\subsection{General Case}

Assume that we have identified the conic correspondences $\mathbf{C}_{i} \leftrightarrow \mathbf{C}_{i}^{\prime}, i=1, \ldots, n$, between two planes which are related by a homography represented with a non-singular $3 \times 3$ matrix $\mathbf{H}$. Further, assume that the conics are non-degenerate so that $\operatorname{det} \mathbf{C}_{i} \neq 0, \operatorname{det} \mathbf{C}_{i}^{\prime} \neq 0$. The transformation rule (2) gives

$$
\begin{array}{rc}
s_{1} \mathbf{C}_{1} & =\mathbf{H}^{\top} \mathbf{C}_{1}^{\prime} \mathbf{H} \\
\vdots & \vdots \\
s_{n} \mathbf{C}_{n} & =\mathbf{H}^{\top} \mathbf{C}_{n}^{\prime} \mathbf{H},
\end{array}
$$

where we have explicitly written out the scale factors $s_{i}$ so that we get a set of inhomogeneous equations. This implies that $s_{i}^{3} \operatorname{det} \mathbf{C}_{i}=\operatorname{det} \mathbf{C}_{i}^{\prime}(\operatorname{det} \mathbf{H})^{2}$ for all $i$. Since the matrix $\mathbf{H}$ is defined only up to scale we may fix $(\operatorname{det} \mathbf{H})^{2}=1$ which gives

$$
s_{i}=\left(\frac{\operatorname{det} \mathbf{C}_{i}^{\prime}}{\operatorname{det} \mathbf{C}_{i}}\right)^{1 / 3} .
$$

Hence, without any loss of generality, we may normalize the conics $\mathbf{C}_{i}$ so that $\operatorname{det} \mathbf{C}_{i}=$ $\operatorname{det} \mathbf{C}_{i}^{\prime}$ and it is enough to consider the inhomogeneous equations $\mathbf{C}_{i}=\mathbf{H}^{\top} \mathbf{C}_{i}^{\prime} \mathbf{H}, i=1, \ldots, n$. Consider any two of these equations

$$
\begin{aligned}
\mathbf{C}_{i} & =\mathbf{H}^{\top} \mathbf{C}_{i}^{\prime} \mathbf{H} \\
\mathbf{C}_{j} & =\mathbf{H}^{\top} \mathbf{C}_{j}^{\prime} \mathbf{H}
\end{aligned}
$$

from which we get

$$
\mathbf{C}_{i}^{-1} \mathbf{C}_{j}=\mathbf{H}^{-1} \mathbf{C}_{i}^{\prime-1} \mathbf{C}_{j}^{\prime} \mathbf{H} .
$$

This shows the "only if" part of the following result, and the "if" part will be shown in the following section.

Lemma 1 There is a projective transformation which maps the non-degenerate plane conics $\mathbf{C}_{1}$ and $\mathbf{C}_{2}$ to $\mathbf{C}_{1}^{\prime}$ and $\mathbf{C}_{2}^{\prime}$, respectively, if and only if there exists $\delta \in \mathbb{R}$ so that $\delta \mathbf{C}_{1}^{-1} \mathbf{C}_{2}$ and $\mathbf{C}_{1}^{\prime-1} \mathbf{C}_{2}^{\prime}$ are similar matrices.

We proceed by writing (7) in the form

$$
\mathbf{C}_{i}^{\prime-1} \mathbf{C}_{j}^{\prime} \mathbf{H}-\mathbf{H C}_{i}^{-1} \mathbf{C}_{j}=\mathbf{0}
$$

which is a set of linear equations in the elements of $\mathbf{H}$, i.e.

$$
\mathbf{M}_{i j} \mathbf{h}=\mathbf{0},
$$


where $\mathbf{h}$ is a $9 \times 1$ vector containing the elements of $\mathbf{H}$ and $\mathbf{M}_{i j}$ is a $9 \times 9$ matrix determined by the conics. Thus, the solution $\mathbf{h}$ belongs to the null space of $\mathbf{M}_{i j}$. However, in general, the dimension of the null space of $\mathbf{M}_{i j}$ is greater than 1. This is because (7) does not determine $\mathbf{H}$ uniquely. Nevertheless, if $n \geq 3$ we may choose other two equations to get another set of linear constraints. By considering all ordered pairs, we have in total $n(n-1)$ pairs of equations and by stacking the matrices $\mathbf{M}_{i j}$ we get an overdetermined set of $9 n(n-1)$ equations

$$
\mathbf{M h}=\mathbf{0}
$$

so that the null space is usually one dimensional. We found that, in general, already three conic correspondences allow to solve $\mathbf{h}$ from (10). In practice, $\mathbf{M}$ may have full rank due to measurement errors in the conic coefficients but in this case the solution minimizing $\|\mathbf{M h}\|$ with $\|\mathbf{h}\|=1$ is obtained as the singular vector corresponding to the smallest singular value of $\mathbf{M}$ [3]. We get the solution $\mathbf{h}$ up to scale and, if desired, we may scale the elements so that $\operatorname{det} \mathbf{H}=1$. Thus, we have a linear algorithm for solving $\mathbf{H}$ from $n$ conic correspondences when $n \geq 3$.

\subsection{Minimal Case: Two Correspondences}

Given two conic correspondences, the transformation rule (2) gives a pair of quadratic matrix equations for $\mathbf{H}$, as discussed above. The solvability of these equations was addressed in Lemma 1. In fact, it is well known that for a pair of plane conics $\mathbf{C}_{1}, \mathbf{C}_{2}$ the eigenvalues of $\mathbf{C}_{1}^{-1} \mathbf{C}_{2}$ are projectively invariant up to scale [2]. Thus, these invariants provide a necessary and sufficient condition for the existence of a solution. However, since the solution is not unique, i.e. not unique even up to scale, the linear algorithm of the previous section may not provide a good solution. Hence, we propose here another algorithm.

We may proceed as in the previous section and fix $(\operatorname{det} \mathbf{H})^{2}=1$ and $\operatorname{det} \mathbf{C}_{i}=\operatorname{det} \mathbf{C}_{i}^{\prime}$ for $i=1,2$, so that we obtain the inhomogeneous equations

$$
\begin{aligned}
& \mathbf{C}_{1}=\mathbf{H}^{\top} \mathbf{C}_{1}^{\prime} \mathbf{H}, \\
& \mathbf{C}_{2}=\mathbf{H}^{\top} \mathbf{C}_{2}^{\prime} \mathbf{H} .
\end{aligned}
$$

Next, we will use the following factorization for symmetric matrices.

Lemma 2 If $\mathbf{C}$ is a real symmetric matrix then $\mathbf{C}=\mathbf{F F}^{\top}$ for some matrix $\mathbf{F}$ which may be complex. If $\mathbf{C}$ is invertible and $\mathbf{C}=\mathbf{F} \mathbf{F}^{\top}=\mathbf{F}_{1} \mathbf{F}_{1}^{\top}$, then $\mathbf{F}_{1}=\mathbf{F R}$ where $\mathbf{R}$ is a complex orthogonal matrix, $\mathbf{R}^{\top} \mathbf{R}=\mathbf{I}$.

Proof: In the appendix.

We use this factorization and write (11) as

$$
\mathbf{F}_{1} \mathbf{F}_{1}^{\top}=\mathbf{H}^{\top} \mathbf{F}_{1}^{\prime} \mathbf{F}_{1}^{\prime \top} \mathbf{H}
$$

which gives $\mathbf{F}_{1}=\mathbf{H}^{\top} \mathbf{F}_{1}^{\prime} \mathbf{R}$ where $\mathbf{R}^{\top} \mathbf{R}=\mathbf{I}$. Thus $\mathbf{H}=\mathbf{F}_{1}^{\prime-\top} \mathbf{R} \mathbf{F}_{1}^{\top}$, and substituting this to (12) gives a pair of equations which is equivalent to the original one:

$$
\begin{aligned}
\mathbf{R}^{\top} \mathbf{R} & =\mathbf{I}, \\
\mathbf{R}^{\top} \mathbf{A} \mathbf{R} & =\mathbf{B},
\end{aligned}
$$


where $\mathbf{A}=\mathbf{F}_{1}^{\prime-1} \mathbf{C}_{2}^{\prime} \mathbf{F}_{1}^{\prime-\top}$ and $\mathbf{B}=\mathbf{F}_{1}^{-1} \mathbf{C}_{2} \mathbf{F}_{1}^{-\top}$ are complex symmetric matrices. Now, if (14), (15) has a solution then $\mathbf{A}$ is similar to $\mathbf{B}$. Conversely, if $\mathbf{A}$ is similar to $\mathbf{B}$ then by [4, Section 4.4] the matrices are similar via a complex orthogonal matrix, which shows that (14), (15) has a solution. By noticing that $\mathbf{A}$ is similar to $\mathbf{C}_{1}^{\prime-1} \mathbf{C}_{2}^{\prime}$ and $\mathbf{B}$ is similar to $\mathbf{C}_{1}^{-1} \mathbf{C}_{2}$, we see that (11), (12) has a solution if $\mathbf{C}_{1}^{-1} \mathbf{C}_{2}$ is similar to $\mathbf{C}_{1}^{\prime-1} \mathbf{C}_{2}^{\prime}$. This shows the "if" part of Lemma 1.

It remains to find an $\mathbf{R}$ which satisfies (14), (15). If $\mathbf{A}$ (or $\mathbf{B}$ ) has a multiple eigenvalue then there may be infinitely many solutions (these solutions can be characterized using Lemma 3 in the appendix if $\mathbf{A}$ is diagonalizable). For example, if $\mathbf{C}_{1}$ and $\mathbf{C}_{2}$ are two concentric circles their projection does not determine the homography since the circles are invariant to rotations around their centre. We are mainly interested in cases where there are only finitely many solutions, and thus in the following we assume that $\mathbf{A}$ and $\mathbf{B}$ have distinct eigenvalues.

When $\mathbf{A}$ and $\mathbf{B}$ have distinct eigenvalues they are diagonalizable and, in particular, complex orthogonally diagonalizable [4]. By computing their eigenvalues and eigenvectors we get factorizations $\mathbf{A}=\mathbf{Q D Q}{ }^{\top}$ and $\mathbf{B}=\mathbf{U D U}^{\top}$, where $\mathbf{Q}$ and $\mathbf{U}$ are complex orthogonal and $\mathbf{D}$ is diagonal (since $\mathbf{A}$ and $\mathbf{B}$ are similar we may arrange the diagonal factors to be the same). Then (15) implies

$$
\mathbf{R}^{\top} \mathbf{Q D Q}{ }^{\top} \mathbf{R}=\mathbf{U D U}^{\top}
$$

and Lemma 3 in the appendix gives $\mathbf{R}^{\top} \mathbf{Q}=\mathbf{U P}$ where $\mathbf{P}=\operatorname{diag}( \pm 1, \pm 1, \pm 1)$. Thus $\mathbf{R}=\mathbf{Q P U}^{\top}$ and

$$
\mathbf{H}=\mathbf{F}_{1}^{\prime-\top} \mathbf{Q P} \mathbf{U}^{\top} \mathbf{F}_{1}^{\top}
$$

is a solution to (11),(12). Moreover, any solution is of this form. Notice that $\mathbf{H}$ in (17) satisfies $(\operatorname{det} \mathbf{H})^{2}=1$ since $\operatorname{det} \mathbf{C}_{1}=\operatorname{det} \mathbf{C}_{1}^{\prime}$. However, since $\mathbf{H}$ may be multiplied with \pm 1 without changing the actual homography it is enough to consider only four of the above eight choices for $\mathbf{P}$. Hence, in general, the original homogeneous equations have four solutions of which some may be complex. If we have a nonreal solution then its complex conjugate is also a solution, which implies that there are 0,2 or 4 nonreal solutions.

Above we have constructed an algorithm for solving a homography from two conic correspondences. There exists a four-fold ambiguity of solutions. The geometrically correct homography can be chosen if, for example, a point correspondence is known. The proposed algorithm involves only linear algebra: the factorization of Lemma 2 and the factorization of $\mathbf{A}$ and $\mathbf{B}$ require the eigenvalues and eigenvectors of $3 \times 3$ symmetric matrices and nothing else.

\subsection{Implementation}

We summarize the proposed algorithms in the following.

\section{Algorithm 1: homography from $n$ conic correspondences}

(i) Given the conic correspondences normalize all the coefficient matrices to have unit Frobenius norm. Denote the obtained matrices by $\mathbf{C}_{i}, \mathbf{C}_{i}^{\prime}, i=1, \ldots, n$. Then, for all $i$, replace $\mathbf{C}_{i}$ with $s_{i} \mathbf{C}_{i}$ where $s_{i}$ is given in (4).

(ii) For each ordered pair $\{i, j\}, i, j=1, \ldots, n$, compute $\mathbf{C}_{i}^{-1} \mathbf{C}_{j}$ and $\mathbf{C}_{i}^{\prime-1} \mathbf{C}_{j}^{\prime}$ and use these to form the matrices $\mathbf{M}_{i j}$ (see (8) and (9)). 
(iii) Stack all the matrices $\mathbf{M}_{i j}$ to a $9 n(n-1) \times 9$ matrix $\mathbf{M}$ and compute its singular value decomposition $\mathbf{M}=\mathbf{U S V}^{\top}$ so that the singular values are in descending order on the diagonal of $\mathbf{S}$. Set $\mathbf{h}$ to be the last column of $\mathbf{V}$.

(iv) The vector $\mathbf{h}$ contains the elements of the homography matrix $\mathbf{H}$ (if the configuration of conics is such that they determine the homography uniquely).

\section{Algorithm 2: homography from 2 conic correspondences}

(i) Given the conic correspondences normalize all the coefficient matrices to have unit Frobenius norm. Denote the obtained matrices by $\mathbf{C}_{i}, \mathbf{C}_{i}^{\prime}, i=1,2$. Then, for all $i$, replace $\mathbf{C}_{i}$ with $s_{i} \mathbf{C}_{i}$ where $s_{i}$ is given in (4).

(ii) Compute the eigendecompositions $\mathbf{C}_{1}=\mathbf{V}_{1} \mathbf{D}_{1} \mathbf{V}_{1}^{\top}$ and $\mathbf{C}_{1}^{\prime}=\mathbf{V}_{1}^{\prime} \mathbf{D}_{1}^{\prime} \mathbf{V}_{1}^{\prime \top}$ and set $\mathbf{F}_{1}=\mathbf{V}_{1} \mathbf{D}_{1}^{1 / 2}$ and $\mathbf{F}_{1}^{\prime}=\mathbf{V}_{1}^{\prime} \mathbf{D}_{1}^{\prime 1 / 2}$.

(iii) Compute $\mathbf{A}=\mathbf{F}_{1}^{\prime-1} \mathbf{C}_{2}^{\prime} \mathbf{F}_{1}^{\prime-\top}$ and $\mathbf{B}=\mathbf{F}_{1}^{-1} \mathbf{C}_{2} \mathbf{F}_{1}^{-\top}$ and their eigendecompositions $\mathbf{A}=\mathbf{Q} \mathbf{D}_{\mathrm{A}} \mathbf{Q}^{\top}$ and $\mathbf{B}=\mathbf{U D}_{\mathrm{B}} \mathbf{U}^{\top}$ where $\mathbf{D}_{\mathrm{A}}=\operatorname{diag}\left(a_{1}, a_{2}, a_{3}\right)$ and $\mathbf{D}_{\mathrm{B}}=$ $\operatorname{diag}\left(b_{1}, b_{2}, b_{3}\right)$.

(iv) Go through all six possible permutations of the eigenvalues $b_{k}$ and compute distances $\sum_{k}\left|a_{k}-b_{k}\right|^{2}$. Choose the ordering that gives the smallest distance and permute the eigenvectors correspondingly to get a new $\mathbf{U}$ (due to measurement errors $\mathbf{A}$ and $\mathbf{B}$ may not be exactly similar).

(v) Set $\mathbf{P}_{1}=\operatorname{diag}(1,1,1), \mathbf{P}_{2}=\operatorname{diag}(-1,1,1), \mathbf{P}_{3}=\operatorname{diag}(1,-1,1)$ and finally $\mathbf{P}_{4}=$ $\operatorname{diag}(1,1,-1)$. The four solutions are $\mathbf{H}_{k}=\mathbf{F}_{1}^{\prime-\top} \mathbf{Q} \mathbf{P}_{k} \mathbf{U}^{\top} \mathbf{F}_{1}^{\top}, k=\{1,2,3,4\}$. In general, one of these solutions should be the geometrically correct one.

In the next section we will show that these algorithms are practical also when there is no exact solution to (3) due to noise and measurement errors in conic coefficients. Related to this we would like to point out an implementational issue. We experimentally found that the selection of coordinate origin and scale has an effect to the solution produced by Algorithm 1 when the conics are obtained by fitting them to noisy points. Hence, we used a similar normalization scheme as in [3, p. 109] for solving a homography from point correspondences. That is: (i) all the points in both images are normalized such that their centroid is the origin and their average distance from the origin is $\sqrt{2}$, (ii) conics are fitted to the normalized points, (iii) homography is solved with Algorithm 1, (iv) the obtained homography is denormalized (see [3] for details).

\section{Experiments}

\subsection{A Simple Example}

First, we illustrate the solutions obtained by the Algorithm 2 with exact data. We constructed two conic coefficient matrices, $\mathbf{C}_{1}$ and $\mathbf{C}_{2}$, so that the first conic is the unit circle and the second is the parabola $y=x^{2}$. Then we applied a rotation of angle $\pi / 2$ about the origin to get $\mathbf{C}_{1}^{\prime}$ and $\mathbf{C}_{2}^{\prime}$, see Fig. 1. Applying the Algorithm 2 provides four solutions of which two are complex. We discarded the complex solutions and transformed the four corner points of a square using the real solutions. From Fig. 1 we can observe that in this case both real solutions are geometrically meaningful due to symmetry, the other is a pure rotation and the other is a rotation combined with a reflection. 


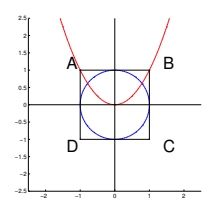

(a)

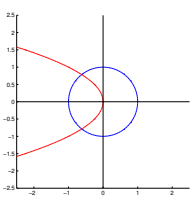

(b)

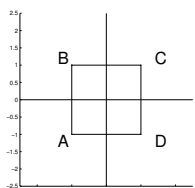

(c)

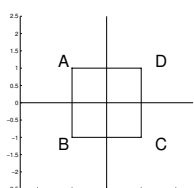

(d)

Figure 1: (a) two conics and a square, (b) the rotated conics, (c) the square transformed with the first real solution (rotation), and (d) with the other real solution (rotation and reflection)

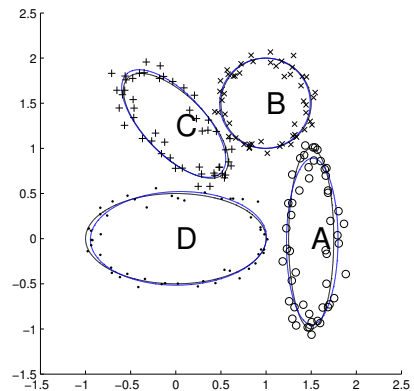

(a)

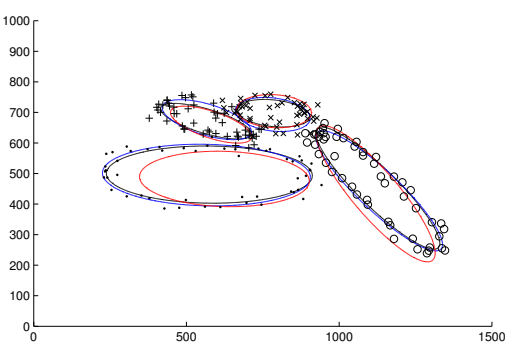

(b)

Figure 2: Two patterns of four ellipses. The ground truth ellipses are in black and the fitted ellipses in blue. Each ellipse was fitted to 50 points perturbed with Gaussian noise. Here the standard deviation of noise is approximately $2 \%$ of data spread in both images. The ellipses from the first image were transformed to the second using the estimated homography, the result is in red.

\subsection{Synthetic Data with Noise}

Next we experimented our algorithms with synthetic data in order to evaluate their sensitivity to noise. In Fig. 2(a) there are two planar patterns consisting of four ellipses. The ground truth homography between the patterns was constructed so that the second pattern would be a result of imaging the first pattern with a typical perspective camera. Notice the different scales between the images. The original ellipses in the first pattern and their exact images in the second pattern are plotted in black. We sampled 50 points uniformly from each ellipse and added Gaussian noise to these points with varying standard deviation. The standard deviation of the noise was proportional to the spread of the data points and varied between 0 and $2 \%$ of data spread. The measured conic coefficients were obtained by fitting ellipses to the noisy points using the method [1]. The fitted ellipses are plotted in blue.

We estimated the homography at different levels of noise using two (A and B), three (A, B and C) or four ellipse correspondences. At each noise level we did 1000 trials involving ellipse fitting and homography estimation. The estimated homography was compared to the ground truth by mapping the four points $(1,1),(1,-1),(-1,-1)$, and $(-1,1)$ from the first image to the second image with both homographies and then computing the root-mean-squared (RMS) distance of the corresponding points in the second image. In Fig. 3 we have plotted the average values of the logarithm of this RMS distance among the 1000 trials for six different homography estimates. Among the six estimates we have also the estimate from four point correspondences, computed using the normal- 


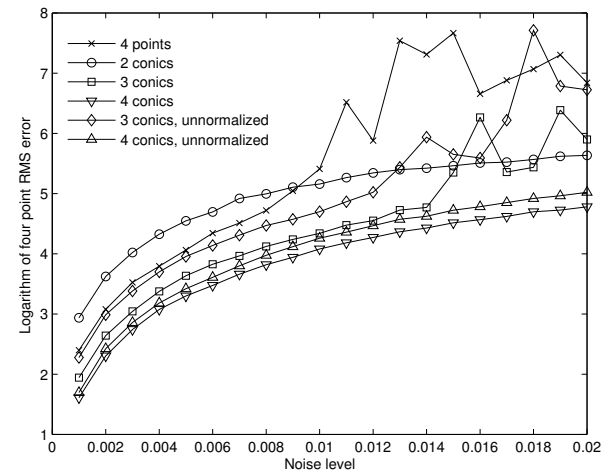

Figure 3: The average estimation error among 1000 trials at each noise level. Notice the logarithmic scale. The experimental set-up is illustrated in Fig. 2.

ized DLT method [3, p. 109]. Here we used the centres of ellipses A, B, C and D together with their projections in the second image as the four point correspondences. These points were perturbed with the same Gaussian noise as the points on the ellipses.

It can be seen from Fig. 3 that the conic based methods are here more robust than the point based method. This is expected since there are only four point correspondences while there are 50 points per ellipse. It can also be seen that the robustness to noise increases while the number of conic correspondences increases. Further, the normalization of image coordinates that was discussed in Section 3.3 improves the results when used together with Algorithm 1. This is analogous to the point based method [3]. However, we found that the normalization of image coordinates had no effect on the result of Algorithm 2 in this experiment. In addition, we experimented whether it is really useful to consider all ordered pairs of conics in the step (ii) of Algorithm 1 or would it be sufficient to take each pair only once. Our observation was that using all ordered pairs gives better robustness to noise.

\subsection{Real Data}

We did experiments also with real images. In Fig. 4 we have two views of a plane containing white circles on black background. The ellipses, i.e. imaged circles, were detected by first thresholding the images and then fitting ellipses to the boundary points of the binary blobs using [1]. The detected ellipses are shown in cyan. The homography between the views was estimated using 2 and 36 ellipse correspondences. The results are shown in Figs. 4(b) and 4(c), respectively. It can be seen that when only two ellipse correspondences are used (those in the right hand side corners of the second image) there is a notable registration error. However, we found that if we would use the two ellipses in the lower left and upper right corners the registration result would be visually the same as when using all the ellipses. This indicates that two ellipse correspondences are sufficient to recover the homography with a reasonable accuracy also in practice. 


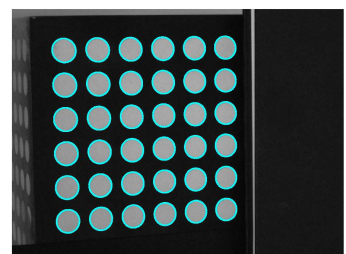

(a)

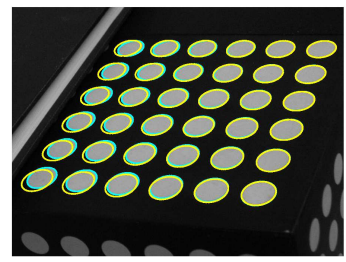

(b)

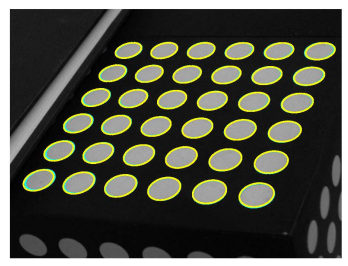

(c)

Figure 4: (a) Image of a plane containing white circles. The detected ellipses are in cyan. (b) Another view. The homography was estimated using the two ellipses in the right upper and lower corners. The ellipses transformed from the first view are in yellow. (c) The homography estimated using all the ellipses.

\section{Conclusions}

We have proposed two new algorithms for computing a planar homography from conic correspondences. The first algorithm can be used when there are at least three conic correspondences and, in general, it provides a unique solution up to scale. The second algorithm is for the minimal case of two conic correspondences and, in general, it provides a solution up to a four-fold ambiguity. Both algorithms require only linear algebra and they are easy to implement as described in Section 3.3. In the experiments we showed that the proposed algorithms provide a reasonable estimate of the homography also when no exact solution exists due to measurement errors in the conic coefficients.

\section{Aknowledgements}

The authors would like to thank Sami Brandt and Esa Rahtu for fruitful discussions.

\section{A Appendix}

Proof of Lemma 2: The spectral theorem says that $\mathbf{C}=\mathbf{V D V}^{\top}$ where $\mathbf{V}$ is real orthogonal and $\mathbf{D}$ is real diagonal. Hence, taking $\mathbf{F}=\mathbf{V D}^{1 / 2}$ gives $\mathbf{C}=\mathbf{F} \mathbf{F}^{\top}$. Note that $\mathbf{D}^{1 / 2}$ will be complex if $\mathbf{D}$ contains negative diagonal elements. If $\mathbf{C}$ is invertible and $\mathbf{C}=\mathbf{F F}{ }^{\top}=\mathbf{F}_{1} \mathbf{F}_{1}^{\top}$ then $(\operatorname{det} \mathbf{F})^{2}=\left(\operatorname{det} \mathbf{F}_{1}\right)^{2} \neq 0$ so $\mathbf{F}$ and $\mathbf{F}_{1}$ are invertible. We get $\mathbf{F}_{1}=\mathbf{F R}$ for $\mathbf{R}=\mathbf{F}^{-1} \mathbf{F}_{1}$, and

$$
\mathbf{R R}^{\top}=\mathbf{F}^{-1}\left(\mathbf{F}_{1} \mathbf{F}_{1}^{\top}\right) \mathbf{F}^{-\top}=\mathbf{F}^{-1}\left(\mathbf{F F}^{\top}\right) \mathbf{F}^{-\top}=\mathbf{I}
$$

Lemma 3 Let $\mathbf{A}$ be a diagonalizable complex symmetric $3 \times 3$ matrix. Then there exist complex matrices $\mathbf{Q}$ and $\mathbf{D}$, with $\mathbf{Q}^{\top} \mathbf{Q}=\mathbf{I}$ and $\mathbf{D}$ diagonal, such that $\mathbf{A}=\mathbf{Q D Q}^{\top}$. Further, if $\mathbf{D}=\operatorname{diag}\left(\lambda_{1}, \lambda_{2}, \lambda_{3}\right)$ and if $\mathbf{A}=\mathbf{Q}_{1} \mathbf{D} \mathbf{Q}_{1}^{\top}$ is another factorization with $\mathbf{Q}_{1}^{\top} \mathbf{Q}_{1}=\mathbf{I}$, then $\mathbf{Q}_{1}=\mathbf{Q P}$ where $\mathbf{P}$ has the following form.

1. If $\lambda_{1}, \lambda_{2}, \lambda_{3}$ are distinct then $\mathbf{P}=\operatorname{diag}( \pm 1, \pm 1, \pm 1)$.

2. If $\lambda_{1}=\lambda_{2} \neq \lambda_{3}$ then $\mathbf{P}=\left(\begin{array}{cc}\mathbf{U} & 0 \\ 0 & \pm 1\end{array}\right)$ where $\mathbf{U}$ is a complex $2 \times 2$ matrix with $\mathbf{U}^{\top} \mathbf{U}=\mathbf{I}$. 
3. If $\lambda_{1}=\lambda_{2}=\lambda_{3}$ then $\mathbf{A}$ is diagonal and $\mathbf{P}$ can be any complex orthogonal matrix.

Proof: The existence of a factorization $\mathbf{A}=\mathbf{Q D Q} \mathbf{Q}^{\top}$ follows from the fact that $\mathbf{A}$ is diagonalizable and [4, Section 4.4]. Let $\mathbf{A}=\mathbf{Q}_{1} \mathbf{D} \mathbf{Q}_{1}^{\top}$ be another such factorization, and write $\mathbf{q}_{j}$ and $\tilde{\mathbf{q}}_{j}$ for the columns of $\mathbf{Q}$ and $\mathbf{Q}_{1}$, respectively. If all $\lambda_{j}$ are distinct, then each eigenspace is one dimensional and orthogonality implies $\tilde{\mathbf{q}_{j}}= \pm \mathbf{q}_{j}$. If $\lambda_{1}=\lambda_{2} \neq \lambda_{3}$, then the first eigenspace is two dimensional and

$$
\left(\begin{array}{lll}
\tilde{\mathbf{q}}_{1} & \tilde{\mathbf{q}}_{2} & \tilde{\mathbf{q}}_{3}
\end{array}\right)=\left(\begin{array}{lll}
\mathbf{q}_{1} & \mathbf{q}_{2} & \mathbf{q}_{3}
\end{array}\right)\left(\begin{array}{cc}
\mathbf{U} & 0 \\
0 & \pm 1
\end{array}\right)
$$

for some complex $2 \times 2$ matrix $\mathbf{U}$. Orthogonality implies $\mathbf{U}^{\top} \mathbf{U}=\mathbf{I}$. The case where all eigenvalues are equal is obvious.

\section{References}

[1] A. W. Fitzgibbon, M. Pilu, and R. B. Fisher. Direct least square fitting of ellipses. TPAMI, 21(5):476-480, 1999.

[2] D. Forsyth, J. L. Mundy, A. Zisserman, C. Coelho, A. Heller, and C. Rothwell. Invariant descriptors for 3-D object recognition and pose. TPAMI, 13(10), 1991.

[3] R. Hartley and A. Zisserman. Multiple View Geometry in Computer Vision. Cambridge, 2nd edition, 2003.

[4] R. A. Horn and C. R. Johnson. Matrix Analysis. Cambridge, 1985.

[5] F. Kahl and A. Heyden. Using conic correspondences in two images to estimate the epipolar geometry. In ICCV, 1998.

[6] J. Y. Kaminski and A. Shashua. Multiple view geometry of general algebraic curves. IJCV, 56(3):195-219, 2004.

[7] P. Mudigonda, C. V. Jawahar, and P. J. Narayanan. Geometric structure computation from conics. In ICVGIP, 2004.

[8] L. Quan. Conic reconstruction and correspondence from two views. TPAMI, 18(2):151-160, February 1996.

[9] C. Schmid and A. Zisserman. The geometry and matching of lines and curves over multiple views. IJCV, 40(3):199-233, 2000.

[10] J. G. Semple and G. T. Kneebone. Algebraic Projective Geometry. Oxford, 1952.

[11] A. Sugimoto. A linear algorithm for computing the homography from conics in correspondence. Journal of Mathematical Imaging and Vision, 13:115-130, 2000.

[12] C. Yang, F. Sun, and Z. Hu. Planar conic based camera calibration. In ICPR, 2000. 